Vilma Guimarães de Mendonça ${ }^{1}$

Felipe Rinald Barbosa LoRenZato ${ }^{2}$

Juliana Guimarães de MENDONÇA ${ }^{3}$

Telma Cursino de Menezes ${ }^{1}$

Maria José BezerRa Guimarães ${ }^{4}$

\title{
Mortalidade por câncer do colo do útero: características sociodemográficas das mulheres residentes na cidade de Recife, Pernambuco
}

\author{
Uterus cervix cancer mortality: socio-demographic characteristics \\ of women living in the city of Recife, Pernambuco, Brazil
}

Fatores socioeconômicos

Dados demográficos Indicadores básicos de saúde Mortalidade

Neoplasias do colo do útero/ mortalidade

Neoplasias uterinas/mortalidade

Estudos transversais

Keywords

Socioeconomic factors Demographic data

Health status indicators

Mortality

Uterine cervical neoplasms/ mortality

Uterine neoplasms/mortality

Cross-sectional studies

\section{Resumo}

OBJETIVO: descrever as características sociodemográficas dos óbitos por câncer do colo uterino em residentes de Recife, Pernambuco, ocorridos entre 2000 e 2004. MÉTODOS: realizou-se um estudo transversal, populacional, com a inclusão de 323 óbitos por câncer do colo uterino, dos quais 261 encontravam-se registrados no Sistema de Informação sobre Mortalidade e 62 foram identificados após investigação das mortes por câncer em porção não especificada do útero. Obteve-se a mortalidade proporcional para todas as variáveis e foram calculadas medidas estatísticas de tendência central e dispersão para a idade. Quanto ao local de residência e faixa etária, realizou-se o teste do $\chi^{2}$ e obteve-se o coeficiente de mortalidade. RESULTADOS: houve predomínio de óbitos em mulheres com idade inferior a 60 anos $(54,7 \%)$, negras $(60,5 \%)$, sem companheiro $(67,7 \%)$, donas de casa $(71,2 \%)$ e residentes em bairros com baixa condição de vida $(53,3 \%)$. A maioria das mortes foi hospitalar $(85,1 \%)$, das quais $90,2 \%$ ocorreram na rede do Sistema Único de Saúde (SUS). O coeficiente de mortalidade variou de 0,3 (na faixa etária inferior a 30 anos) a 54,9/100.00 (em maiores de 80 anos). Comparando-se os óbitos por faixa etária e distrito sanitário com as características da população feminina residente na cidade, evidenciaram-se diferenças estatisticamente significantes $(p<0,05)$. CONCLUSÕES: em Recife, os óbitos por câncer do colo uterino são mais freqüentes em mulheres na idade adulta, negras, sem companheiro, donas de casa, residentes em bairros com baixa condição de vida e atendidas na rede hospitalar do SUS, existindo diferenças no risco de morte entre as faixas etárias e o local de residência.

\section{Abstract}

PURPOSE: to describe the socio-demographic characteristics of deaths caused by uterine cervix cancer in women living in Recife, Pernambuco, Brazil, from 2000 to 2004. METHODS: a transversal populational study, including 323 deaths by uterine cervix cancer, among which 261 were recorded in the Information System about Mortality and 62 were identified after investigation on deaths by cancer at non-specified sites of the uterus. Mortality rate for all the variables was obtained and statistics for central tendency and variance were calculated. The $\chi^{2}$ test was performed to obtain the mortality coefficient concerning the living place and age range of the patients. RESULTS: death among women under $60(54.7 \%)$, black (60.5\%), single (67.6\%), housewives (71.2\%) and the ones living in poor neighborhood (53.3\%) preponderated. Most of deaths occurred in hospitals (85.1\%) and $90.2 \%$ of them occurred inside national health system hospitals. The mortality coefficient varied from 0.3 (among women under 30) to 54.9/100.00 (among women over $80)$. Significant statistical differences $(p<0.05)$ were evidenced when death linked to age range and sanitary district was compared to characteristics of the female population living in the city. CONCLUSIONS: in Recife, death by cervix cancer are more frequent among adult, black, single, housewives, women living in poor neighborhoods and attended to at national health system hospitals, with differences in death risk among age ranges and living place.
Correspondência:

Vilma Guimarães de Mendonça. Rua Ferreira Lopes, 401/2.102 - Parnamirim CEP: $52060-200$ - Recife/PE

Fone: (81) 3268-2978 E-mail: vilmagui@hotmail.com

Recebido

$6 / 3 / 08$

Aceito com modificacõos
Instituto Materno Infantil Professor Fernando Figueira - IMIP - Recife (PE), Brasil.

' Preceptora da Residência Médica em Ginecologia e Obstetrícia do Instituto Materno Infantil Professor Fernando Figueira - IMIP Recife (PE), Brasil.

2 Professor do Programa de Pós-graduação do Instituto Materno Infantil Professor Fernando Figueira - IMIP - Recife (PE), Brasil. ${ }^{3}$ Acadêmica de Medicina da Universidade Federal de Pernambuco - UFPE - Recife (PE). Brasil; Colaboradora do Programa Institucional de Bolsas de Iniciação Científica do Conselho Nacional de Desenvolvimento Científico e Tecnológico - Pibic/CNPq ${ }^{4}$ Epidemiologista da Secretaria de Saúde do Recife - Recife (PE), Brasil. 


\section{Introdução}

O câncer do colo do útero, quando diagnosticado e tratado precocemente, constitui uma causa de morte evitável. O diagnóstico precoce e o tratamento eficaz da doença são viáveis, pois esta apresenta etapas bem definidas, longo período para a evolução das lesões precursoras e facilidade de detecção das alterações na fase inicial, o que lhe confere um dos mais altos potenciais de prevenção e cura entre todos os tipos de câncer ${ }^{1,2}$.

Representa o terceiro câncer mais comum no mundo, estimando-se o diagnóstico de mais de 471.000 casos novos anuais, dos quais cerca de $80 \%$ ocorrem em países em desenvolvimento. Em algumas regiões, é o câncer mais freqüente entre as mulheres, como no sul e leste da África, América Central, centro-sul da Ásia e na Melanésia ${ }^{3}$.

No Brasil, segundo estimativas do Instituto Nacional do Câncer (INCA), espera-se 18.680 casos novos de câncer do colo do útero em 2008, correspondendo a um risco estimado de 19,2/100.000 mulheres. Nas diferentes regiões do país, a incidência da doença é heterogênea: 24,4/100.000 mulheres no Sul, 22,2 no Norte, 19,4 no Centro-Oeste, 17,8 no Sudeste e 17,6 no Nordeste. Ainda para 2008, entre os Estados nordestinos, estima-se a mais alta incidência para Sergipe $(24,0)$ e a mais baixa para a Paraíba (12,2). Em Pernambuco, são esperados 1.020 casos novos (22,7/100.000 mulheres) e, em Recife, 210 casos novos (25,6/100.000 mulheres), representando o segundo câncer mais incidente em mulheres no Estado e na capital, excluindo-se os tumores malignos de pele não melanoma ${ }^{4}$.

O câncer do colo do útero acomete mulheres na faixa etária reprodutiva, concentrando-se naquelas com idade acima de 35 anos, com pico máximo de incidência entre 45 e 49 anos. No entanto, tem sido observado um aumento da ocorrência em mulheres mais jovens ${ }^{5}$. É mais freqüente em mulheres de populações urbanas, de classe social e escolaridade mais baixas, residentes em países em desenvolvimento, negras, não virgens, multíparas, com início precoce de relações sexuais, primeira gestação em idade jovem, múltiplos parceiros e fumantes ${ }^{6}$. Acredita-se que a infecção pelo papilomavírus humanos (HPV) seja a causa primária do câncer do colo do útero. Sua prevalência na lesão do colo é superior a $98 \%$ e dois subtipos do vírus (16 e 18) estão presentes em mais de $80 \%$ dos casos de câncer invasor. Também são relacionados como co-fatores outras doenças sexualmente transmissíveis, especialmente a presença do vírus da imunodeficiência humana (HIV), o uso de tratamento imunossupressivo e história de transplante de órgãos ${ }^{7}$.

Países em desenvolvimento apresentam altos coeficientes de mortalidade, com óbitos em plena idade produtiva, o que penaliza a mulher e o grupo que lhe é próximo, além de privar a sociedade do seu potencial econômico e intelectual ${ }^{2}$. Aos anos potenciais de vida improdutivos e perdidos decorrentes das mortes precoces por câncer do colo do útero, associam-se, também, o tempo gasto com a própria doença e o sofrimento físico e emocional das mulheres. No Brasil, as taxas de mortalidade ainda são elevadas, com a doença persistindo como um problema de Saúde Pública ${ }^{8}$.

A efetividade da detecção precoce do câncer do útero por meio do exame Papanicolaou, associada ao tratamento da lesão intra-epitelial, tem resultado em uma redução da incidência do câncer invasor do colo do útero de $90 \%$, produzindo um impacto significativo nas taxas de morbimortalidade. Entretanto, segundo recomendação da Organização Pan-Americana de Saúde (OPAS) e da Organização Mundial de Saúde (OMS), um padrão de qualidade e uma cobertura de rastreamento de 80 a $85 \%$ da população de risco são necessários para se conseguir este efeito ${ }^{9,10}$.

Com programas de rastreamento eficazes, diversos países nórdicos conseguiram diminuir as taxas de incidência do câncer do colo do útero entre 1986 e 1995, como Islândia (redução de 67\%), Finlândia (75\%), Suécia $(55 \%)$, Dinamarca $(54 \%)$ e Noruega $(34 \%)$, e, conseqüentemente, a mortalidade pela doença ${ }^{9}$. No Brasil, a melhoria da cobertura do exame citológico ainda não foi suficiente para reduzir a mortalidade por câncer do colo do útero em muitas regiões. O diagnóstico tardio ainda é o mais habitual e pode estar relacionado a dificuldades no acesso da população feminina aos serviços e programas de saúde, baixa capacitação dos recursos humanos envolvidos na atenção oncológica, incapacidade do sistema de saúde para absorver a demanda às Unidades de Saúde e dificuldades dos gestores municipais e estaduais em definir e estabelecer um fluxo assistencial com hierarquização dos diversos níveis de atenção ${ }^{10}$.

No país, os estudos epidemiológicos referentes à mortalidade por câncer do colo do útero têm utilizado o Sistema de Informação sobre Mortalidade (SIM), coordenado pelo Ministério da Saúde, como importante fonte de dados $^{11}$. Tais estudos representam um instrumento para a análise dos padrões de ocorrência e evolução da mortalidade em diferentes populações, para avaliação indireta dos programas de rastreamento e para identificação de grupos de mulheres e localidades com maior risco de óbito por câncer do colo do útero - evento este considerado evitável à luz do conhecimento atual ${ }^{12}$. Assim, o presente artigo teve como objetivo descrever as características sociodemográficas dos óbitos por câncer do colo uterino em residentes em Recife, Pernambuco, ocorridos entre 2000 e 2004. Como são raros os estudos com este enfoque no país ${ }^{11}$, pretendeu-se contribuir para o planejamento e adoção de ações de enfrentamento da doença. 


\section{Métodos}

Os dados estudados referem-se ao município de Recife, capital de Pernambuco, situado na região Nordeste do Brasil, cuja área é totalmente urbana, com 94 bairros e seis distritos sanitários (DS). Na composição de sua população, de 1.422.905 habitantes no ano 2000, 53,2\% se autoclassificam como pertencentes à raça/cor negra (5,3\% preta e $47,9 \%$ parda) e $53,5 \%$ são mulheres, das quais $65,2 \%$ encontram-se em idade fértil (entre dez e 49 anos) e $11 \%$ são idosas (idade igual ou superior a 60 anos). Entre as capitais nordestinas, Recife apresenta o segundo maior valor do Índice de Desenvolvimento Humano (IDH, 0,792) referente ao ano 2000. Entretanto, a cidade apresenta uma sociedade marcada por forte desigualdade social e importante dimensão da pobreza ${ }^{13}$.

Realizou-se um estudo tipo corte transversal, de base populacional, sendo inicialmente incluídos todos os óbitos em mulheres residentes em Recife captados pelo SIM, ocorridos entre 2000 e 2004, cujas causas básicas, de acordo com a $10^{a}$ revisão da Classificação Internacional de Doenças (CID-10) ${ }^{14}$, correspondiam a câncer do colo do útero (261 óbitos) e a câncer do útero em porção não especificada (125 óbitos). Estes últimos foram investigados nos prontuários das pacientes em 28 hospitais da região metropolitana de Recife, além de consultas aos arquivos do Serviço de Verificação de Óbito, ao médico assistente e ao Registro de Câncer de Base Populacional da Secretaria Estadual de Saúde de Pernambuco. Com a investigação foram identificados 62 óbitos $(49,6 \%)$ como sendo decorrentes de câncer do colo do útero. Estes óbitos, em conjunto com os constantes no SIM como secundários a câncer do colo uterino, totalizaram 323 mortes, as quais corresponderam à população de estudo.

Para a escolha das variáveis estudadas, levaram-se em consideração a disponibilidade e a completitude das mesmas no banco de dados do SIM, excluindo-se as que apresentaram alta proporção de dados ignorados, como escolaridade, para minimizar a possibilidade de vieses. Assim, foram selecionadas as variáveis: idade, raça/cor, situação conjugal, ocupação, local e hospital de ocorrência do óbito, além do DS e da condição de vida do bairro de residência. Para obtenção desta última variável, foi considerado o Indicador de Condição de Vida (ICV) dos bairros de Recife, desenvolvido por Guimarães et al. ${ }^{15}$, por meio de análise fatorial, a partir de dados do Censo Demográfico 2000 referentes ao abastecimento de água, esgotamento sanitário, coleta de lixo, analfabetismo da população de dez a 14 anos, renda e anos de estudo do responsável pelo domicílio. De acordo com o ICV, a autora agrupou os bairros de Recife, pela técnica de cluster, em quatro estratos: alta, intermediária, baixa e muito baixa condição de vida. No presente estudo, os dois últimos estratos foram considerados em conjunto como os de pior condição de vida.

Os DS de Recife são numerados de I a VI, apresentando características populacionais e sociais distintas. Porém, em todos existem acentuadas desigualdades internas. $\mathrm{O}$ DS I, localizado na região central da cidade e composto por 11 bairros, é o menos populoso, com cerca de 80.000 habitantes. O DS II, situado na parte norte do município, é o que apresenta a maior densidade demográfica, aglomerando 18 bairros. O DS III compreende 29 bairros, com grandes contrastes econômicos e sociais. O DS IV localiza-se a oeste da cidade, com 12 bairros, e o DS V, na parte sudeste, com 16 bairros. Por outro lado, o DS VI, situado na região sul do município, apesar do menor número de bairros (oito), é o mais populoso, com cerca de 350.000 habitantes ${ }^{13}$.

Para a descrição de algumas variáveis, certas categorias constantes na base de dados do SIM foram agrupadas. As mulheres declaradas como casadas ou em união consensual foram consideradas "com companheiro" e as solteiras, viúvas ou separadas judicialmente, "sem companheiro". Em relação à raça/cor, as categorias preta e parda foram agrupadas e descritas como "negra". Para os óbitos hospitalares, considerou-se o fato do estabelecimento de saúde pertencer ou não à rede assistencial do Sistema Único de Saúde (SUS). Os hospitais do SUS foram classificados em públicos, filantrópicos e contratados, conforme os critérios utilizados pelo Ministério da Saúde ${ }^{16}$.

O banco de dados referente aos óbitos por câncer do colo e em porção não especificada do útero, ocorridos entre 2000 e 2004, foi coletado na Secretaria de Saúde do Recife e comparado com o disponibilizado pelo Ministério da Saúde ${ }^{16}$, não se detectando, entre eles, divergência no número de óbitos. Os dados sobre a identificação dos óbitos por câncer em porção não especificada do útero (nome, endereço, idade, local de ocorrência, realização de necrópsia, médico atestante), presentes apenas no banco coletado na Secretaria de Saúde do Recife, foram essenciais para a escolha da fonte de investigação.

Todos os óbitos por câncer do colo do útero - identificados no SIM ou após a investigação daqueles cuja porção do útero não era especificada - tiveram suas variáveis descritas por meio de freqüências (mortalidade proporcional). Para a idade, por ser uma variável contínua, foram obtidas medidas estatísticas de tendência central e dispersão. Para a faixa etária e DS obteve-se, também, o coeficiente de mortalidade (CM). Foi utilizado, como denominador dos $\mathrm{CM}$, a população de mulheres proveniente do Censo Demográfico 2000 e de estimativas para os anos de 2001 a 2004, disponibilizadas pela Secretaria de Saúde do Recife.

Tanto para a faixa etária quanto para o DS de residência, os óbitos por câncer do colo do útero foram comparados 
com um grupo representado pela população de mulheres, por meio do teste do $\chi^{2}$, com nível de significância de 5\%. A partir da população total de mulheres residentes na cidade, no qüinqüênio 2000-2004, obteve-se o número anual médio de mulheres do período (783.369), o qual foi utilizado como grupo de comparação com os óbitos. Para as demais variáveis, esta comparação não foi viável, por não se dispor, para o qüinqüênio estudado, de dados específicos sobre a população de mulheres.

Toda a análise dos dados, além da apresentação tabular, foi realizada com o auxílio dos programas TabWin, Excel e Statistical Package for Social Sciences (SPSS). O estudo foi aprovado pelo Comitê de Ética em Pesquisa em Seres Humanos do Instituto Materno Infantil Professor Fernando Figueira (IMIP) e, para os óbitos referentes ao quinquênio 2000-2004, disponibilizados pela Secretaria de Saúde do Recife, os autores assumiram o compromisso ético de sua utilização para efeito de pesquisa.

\section{Resultados}

No qüinqüênio 2000-2004, ocorreram 323 óbitos por câncer do colo do útero em mulheres residentes em Recife. As medidas estatísticas de tendência central e dispersão dos óbitos mostraram que a distribuição da idade aproximou-se da normalidade, com média e mediana de 57,7 e 57,0 anos, respectivamente, e desvio padrão de 16,2 anos. A idade mínima foi de 16 anos e a máxima de 93 anos, com amplitude de 82 anos, limite do primeiro quartil de 46 anos e do terceiro quartil de 70 anos.
A maioria dos óbitos, conforme a Tabela 1, ocorreu em mulheres com idade inferior a 60 anos $(54,7 \%)$, destacando-se as faixas etárias de 40 a 49 e 50 a 59 anos, com a presença de 20,1 e 20,4\%, respectivamente. Também, foi relevante a ocorrência de óbitos em mulheres entre 30 e 39 anos $(12,4 \%)$ e com idade igual ou inferior a 29 anos $(1,9 \%)$. Ainda em relação à faixa etária, entre os óbitos e a população de mulheres residentes na cidade, observaram-se diferenças estatisticamente significantes $(\mathrm{p}<0,05)$. O CM do município foi 8,2/100.000 mulheres, evidenciando-se um gradiente crescente com a idade, variando de 0,3/100.000 mulheres com menos de 30 anos a 54,9/100.000 mulheres com idade igual ou superior a 80 anos.

Quanto ao DS de residência (Tabela 1), o DS I foi o que apresentou a menor mortalidade proporcional $(8 \%)$ e o DS III, a maior proporção de óbitos $(24,1 \%)$. A comparação entre as distribuições de óbitos e da população de mulheres por DS de residência evidenciou diferenças estatisticamente significantes $(p<0,05)$. Estas diferenças expressam-se nos CM: o risco de morte foi desigual entre os DS, variando entre 6,4/100.000 mulheres (DS IV) e 11,9/100.000 mulheres (DS I). Ressalta-se que o risco de morte em mulheres residentes no DS I e no DS III foi, respectivamente, 1,9 e 1,6 vezes superior ao observado no DS IV.

Conforme a Tabela 2, a maioria dos óbitos ocorreu em mulheres da raça/cor negra $(60,5 \%)$ e que não tinham companheiro $(67,7 \%)$. Salienta-se, quanto à ocupação, que $71,2 \%$ das mulheres eram donas de casa e 9,6\%,

Tabela 1 - Distribuição dos óbitos por câncer do colo do útero, população de mulheres e coeficientes de mortalidade segundo faixa etária e distrito sanitário em residentes em Recife, no qüinqüênio 2000-2004

\begin{tabular}{|c|c|c|c|c|c|c|c|}
\hline \multirow{2}{*}{ Variável } & & \multicolumn{2}{|c|}{ Óbitos } & \multicolumn{2}{|c|}{ População de mulheres* } & \multirow[b]{2}{*}{$\mathbf{p}^{* *}$} & \multirow[b]{2}{*}{$\mathrm{CM}^{* * *}(8,2)$} \\
\hline & & $\begin{array}{c}n \\
(323) \\
\end{array}$ & $\begin{array}{c}\% \\
(100,0) \\
\end{array}$ & $\begin{array}{c}n \\
(783.369) \\
\end{array}$ & $\begin{array}{c}\% \\
(100,0) \\
\end{array}$ & & \\
\hline \multirow{7}{*}{$\begin{array}{l}\text { Faixa etária } \\
\text { (em anos) }\end{array}$} & $\leq 29$ & 6 & 1,9 & 408.049 & 52,1 & \multirow[t]{7}{*}{0,000} & 0,3 \\
\hline & $30-39$ & 40 & 12,4 & 125.489 & 16,0 & & 6,4 \\
\hline & $40-49$ & 65 & 20,1 & 97.991 & 12,5 & & 13,3 \\
\hline & $50-59$ & 66 & 20,4 & 65.340 & 8,3 & & 20,2 \\
\hline & $60-69$ & 64 & 19,8 & 45.733 & 5,8 & & 28,0 \\
\hline & $70-79$ & 48 & 14,9 & 28.378 & 3,6 & & 33,8 \\
\hline & $\geq 80$ & 34 & 10,5 & 12.389 & 1,6 & & 54,9 \\
\hline \multirow{6}{*}{$\begin{array}{l}\text { Distrito sanitário } \\
\text { de residência }\end{array}$} & I (Centro) & 26 & 8,0 & 43.665 & 5,6 & \multirow[t]{6}{*}{0,042} & 11,9 \\
\hline & II (Norte) & 52 & 16,1 & 113.240 & 14,5 & & 9,2 \\
\hline & III (Noroeste) & 78 & 24,1 & 155.604 & 19,9 & & 10,0 \\
\hline & IV (Oeste) & 45 & 13,9 & 139.617 & 17,8 & & 6,4 \\
\hline & V (Sudoeste) & 51 & 15,8 & 135.687 & 17,3 & & 7,5 \\
\hline & VI (Sul) & 71 & 22,0 & 195.556 & 25,0 & & 7,3 \\
\hline
\end{tabular}

(Número): total da coluna.

* Número médio anual da população de mulheres no qüinqüênio 2000-2004;

* * nível descritivo de probabilidade do teste do $\chi^{2}$;

*** $\mathrm{CM}=$ coeficiente de mortalidade por 100.000 mulheres. 
Tabela 2 - Distribuição dos óbitos por câncer do colo do útero segundo variáveis sociodemográficas em residentes em Recife, no qüinqüênio 2000-2004

\begin{tabular}{|c|c|c|c|}
\hline \multicolumn{2}{|c|}{ Variáveis } & $\begin{array}{l}\text { Número } \\
\text { de óbitos* }\end{array}$ & $\begin{array}{c}\% \\
(100,0)\end{array}$ \\
\hline \multirow{3}{*}{ Raça/cor } & Negra & 184 & 60,5 \\
\hline & Branca & 118 & 38,8 \\
\hline & Amarela & 2 & 0,7 \\
\hline \multirow{2}{*}{ Situação conjugal } & Sem companheiro & 210 & 67,7 \\
\hline & Com companheiro & 100 & 32,3 \\
\hline \multirow{9}{*}{ Ocupação } & Dona de casa & 223 & 71,2 \\
\hline & Aposentada & 30 & 9,6 \\
\hline & Empregada doméstica & 9 & 2,9 \\
\hline & Comerciante & 7 & 2,2 \\
\hline & Ajudante de cozinha & 5 & 1,6 \\
\hline & Costureira & 5 & 1,6 \\
\hline & Lavadeira & 4 & 1,3 \\
\hline & Professora & 4 & 1,3 \\
\hline & Outras & 26 & 8,3 \\
\hline \multirow{3}{*}{$\begin{array}{l}\text { Estrato de condição } \\
\text { de vida do bairro de } \\
\text { residência** }\end{array}$} & Melhor & 38 & 11,8 \\
\hline & Intermediária & 113 & 35,0 \\
\hline & Pior & 172 & 53,3 \\
\hline \multirow{2}{*}{ Local de ocorrência } & Hospital & 275 & 85,1 \\
\hline & Domicílio & 48 & 14,9 \\
\hline
\end{tabular}

*Foram excluídos 19 óbitos com raça/cor ignorada (5,9\% do total), 13 óbitos com situação conjugal ignorada $(4 \%)$ e 10 óbitos com ocupação ignorada $(3,1 \%) ; *$ * segundo estratificação de Guimarães et al. ${ }^{15}$ sobre indicador de condição de vida do bairro de residência.

Tabela 3 - Distribuição dos óbitos hospitalares por câncer do colo do útero segundo a natureza do estabelecimento de ocorrência da morte em residentes em Recife, no qüinqüênio 2000-2004

\begin{tabular}{lcc}
\hline Natureza do hospital & Número de óbitos & $\%$ \\
\hline SUS/filantrópico & 163 & 59,3 \\
SUS/público & 79 & 28,7 \\
SUS/contratado & 6 & 2,2 \\
Não SUS & 27 & 9,8 \\
Total & 275 & 100,0 \\
\hline
\end{tabular}

aposentadas. Em relação à condição de vida do local de residência (Tabela 2), observou-se que 53,3\% residiam em bairros considerados com pior situação, $35 \%$ em bairros do estrato intermediário e apenas $11,8 \%$ em bairros pertencentes ao melhor estrato de condição de vida. Quanto ao local do óbito, $85,1 \%$ ocorreram em hospitais.

Entre os óbitos hospitalares (Tabela 3), observou-se a maior proporção de mortes em unidades pertencentes à rede assistencial do SUS $(90,2 \%)$, ressaltando-se que apenas 27 óbitos ocorreram em hospitais que não eram do SUS $(9,8 \%)$. Entre os óbitos hospitalares, destaca-se que $59,3 \%$ deles ocorreram em unidades filantrópicas conveniadas com o SUS.

\section{Discussão}

Para Recife, com os dados da investigação dos óbitos por câncer do útero em porção não especificada foi possível aproximar-se de forma mais fidedigna do número de óbitos por câncer do colo do útero, obtendo-se um CM de 8,2/100.000 mulheres. Na cidade de São Paulo este coeficiente encontra-se estável, entre 6,0 a 8,0/100.000 mulheres ${ }^{12}$. Na América Latina, o CM padronizado pela população mundial por câncer do colo do útero, em 2000, variou de 4,3, em Porto Rico, a 53,5/100.000, no Haiti, sendo, no Brasil, de 11,5/100.000³. Em países da América do Norte, parte ocidental da Europa, Japão e Israel, o CM por câncer de colo do útero varia de 1,0 a 1,5/100.000². Portanto, o coeficiente de mortalidade em residentes em Recife, observado no presente estudo, foi semelhante ao de São Paulo e inferior ao do Brasil, porém, bem superior ao dos países desenvolvidos. Ressalta-se que entre os DS de Recife, os coeficientes variaram bastante, revelando desigualdades intra-urbanas no risco de morte e sugerindo, na determinação de mortes consideradas evitáveis, o papel do acesso desigual das mulheres ao programa de rastreamento do câncer do colo do útero.

$\mathrm{Na}$ América Latina, onde a incidência do câncer do colo do útero é considerada uma das maiores do mundo, representando um grave problema de Saúde Pública, existe um percentual significativo de mulheres que nunca realizaram o exame Papanicolaou - provavelmente aquelas com maior risco. No Brasil, observou-se, no Estado do Paraná, uma evidente diminuição da mortalidade por câncer do colo do útero a partir da implementação do programa de rastreamento, com aumento na cobertura do exame Papanicolaou de 43 para $86 \%$ da população feminina adulta, em um período de apenas cinco anos de incremento do programa ${ }^{17}$.

No presente estudo, ressalta-se que, na faixa etária inferior a 60 anos, concentrou-se mais da metade de todos os óbitos por câncer do colo do útero, fase de vida produtiva do ponto de vista econômico, uma vez que a mulher urbana no Brasil, de acordo com a lei trabalhista vigente, pode ser aposentada aos 60 anos de idade ${ }^{18}$. A morte abaixo dos 60 anos reflete-se nos indicadores "anos produtivos de vida perdidos" e "anos potenciais de vida perdidos por mulher", que, em Salvador, no ano de 1997, foram de 11,6 e 16,9 anos, respectivamente, para o câncer do colo do útero ${ }^{1}$.

Em Recife, destacam-se as faixas etárias de 40 a 49 e de 50 a 59 anos com os maiores percentuais de óbitos, o que pode estar contribuindo sobremaneira para o aumento dos anos produtivos e potenciais de vida perdidos pelas mulheres residentes na cidade. O CM, que reflete o risco de morte, aumentou progressivamente com a faixa etária, atingindo o pico em mulheres com 80 ou mais anos. Este 
achado encontra-se em consonância com o descrito por outros autores ${ }^{2,12}$.

Ressalta-se que o maior percentual de óbitos por câncer do colo do útero $(53,3 \%)$ ocorreu em residentes nos bairros pertencentes aos piores estratos de condição de vida do município, sugerindo o papel da pobreza na determinação da doença e indicando a baixa efetividade das políticas públicas de controle na população de baixo nível socioeconômico. Existe uma ligação entre regiões pouco desenvolvidas e o câncer do colo do útero, medida por meio de indicadores de baixa condição socioeconômica, tais como: reduzida expectativa de vida, poucos médicos, mais crianças com baixo peso ao nascer e mais adultos com tuberculose e $\mathrm{HIV}^{19}$.

Na população urbana do Canadá, no período de 1971 a 1996, o risco de morte por câncer cervical era superior nos bairros mais pobres, apontando a necessidade de melhoria dos programas de prevenção nestes bairros e da oferta dos avanços recentes nas técnicas de rastreamento (citologia em meio líquido, captura híbrida e teste de HPV $)^{20}$. No caso de Recife, os dados indicam que o rastreamento pelo exame de Papanicolaou precisa ser implementado, para que haja impacto na diminuição dos índices de mortalidade da doença, devendo ser priorizadas as áreas com piores condições de vida, antes da implantação de outras estratégias mais dispendiosas, como a vacina contra o HPV.

Não se conhece uma explicação biológica para o fato de mulheres negras apresentarem maior mortalidade por câncer do colo do útero ${ }^{21}$. Provavelmente, fatores socioeconômicos estão contribuindo para a maior ocorrência da doença nesta raça/cor. Daí, no presente estudo, esta variável ter sido usada como uma aproximação da situação socioeconômica. Em 2006, segundo dados da Secretaria de Saúde do Recife ${ }^{22}, 71,4 \%$ dos óbitos por câncer do colo do útero ocorreram em negras, proporção superior à observada neste estudo, no qüinqüênio 2000-2004.

Estudo sobre os fatores associados à não realização do exame Papanicolaou em Pelotas, Rio Grande do Sul ${ }^{23}$ evidenciou, para mulheres sem companheiro, com idade entre 20 e 59 anos, um risco relativo ajustado significativo de 1,85. Em Fortaleza e no Rio de Janeiro, também foi encontrada associação entre a não realização do exame Papanicolaou e a ausência de companheiro ${ }^{24}$. Relação semelhante pode estar acontecendo em Recife, uma vez que a maioria das mulheres que foram a óbito por câncer do colo do útero não tinha companheiro.

Segundo uma revisão sistemática sobre os determinantes e a cobertura do exame de Papanicolaou no Brasil ${ }^{24}$, a não realização do exame associa-se à baixa ou elevada idade, baixa escolaridade, baixo nível socioeconômico, baixa renda familiar, viver sem companheiro, cor parda, uso de contraceptivo oral, ausência de problemas ginecológicos, vergonha ou medo em relação ao exame, não ter realizado consulta médica no ano anterior à pesquisa, dificuldade de acesso à assistência médica, ausência de solicitação médica e não realização do auto-exame das mamas no ano anterior à pesquisa.

É descrita uma forte associação entre a presença de alteração celular epitelial e a escolaridade até o primeiro grau incompleto, sugerindo a falta de conhecimento quanto ao exame de Papanicolaou e os benefícios de fazê-lo rotineiramente, além da ocorrência de outros fatores de risco associados ao câncer do colo do útero, no grupo de pessoas com baixos IDH ${ }^{25}$. Entretanto, apesar de a baixa escolaridade representar um fator de risco para a não realização do exame Papanicolaou, ocorrência de câncer do colo do útero e conseqüente mortalidade, esta variável não pôde ser descrita neste estudo por encontrar-se ignorada para a maioria dos óbitos, representando uma das limitações ao uso de dados secundários.

Quanto à ocupação, a maioria das mulheres (69\%) era dona de casa. Esta proporção é semelhante à encontrada em Santiago, no Chile, onde 66\% das mulheres que morreram por câncer do colo do útero, em 1995, eram donas de casa e com menos de seis anos de escolaridade ${ }^{26}$. Estudo referente ao município de Rio Branco, no Acre, não observou associação significante entre a ocupação de dona de casa e lesões precursoras do câncer do colo em mulheres adolescentes e adultas jovens ${ }^{25}$.

A razão para a permanência da situação de morbimortalidade por câncer do colo do útero está, provavelmente, na ineficiência dos programas de rastreamento em alcançar as mulheres de risco para a doença, aquelas que nunca realizaram o exame ou realizaram há mais de cinco anos, e de garantir um seguimento e tratamento adequado aos casos detectados. O êxito dos programas de prevenção depende da reorganização da assistência clínico-ginecológica, da capacitação dos profissionais de saúde, da continuidade e qualidade das ações de prevenção e do estabelecimento de intervenções mais humanizadas e eqüitativas, que respeitem as diferenças culturais entre as mulheres e sejam focalizadas em eliminar as barreiras e iniqüidades no acesso e utilização dos serviços preventivos ${ }^{19}$.

A mortalidade por câncer de colo de útero apresentase, portanto, como um importante indicador de condições de vida da população e de qualidade da atenção à saúde da mulher. Altas taxas de mortalidade da doença indicam falhas nos serviços de saúde em realizar o diagnóstico precoce do câncer do colo do útero, seu tratamento e acompanhamento adequado. Assim, a situação da mortalidade permite avaliar indiretamente o acesso e a qualidade da assistência à saúde da mulher, subsidiando o planejamento, a gestão, a avaliação de políticas e ações e a análise da situação de saúde e das condições de vida da população, detectando variações geográficas, temporais e entre grupos sociais. 
No Sistema de Informação do Câncer do Colo do Útero (Siscolo), que computa apenas informações de usuárias do SUS, criado a partir de janeiro de 2000 pelo Ministério da Saúde, é possível obter uma série de informações a respeito do programa de rastreamento do câncer do colo do útero no Brasil ${ }^{27}$. De acordo com este sistema, para Recife, a cobertura de exames realizados, tendo como denominador a população de mulheres com 15 anos ou mais, foi de $11,1 \%$ em 2000 e $23,1 \%$ em 2004 . Houve, portanto, um aumento da cobertura em torno de $100 \%$, entre os anos 2000 e 2004, parte decorrente, provavelmente, da melhoria do registro dos dados no sistema.

Conforme a Pesquisa Nacional por Amostra de Domicílios/Saúde (PNAD/Saúde), de 2003, em torno de $70 \%$ das mulheres com mais de 25 anos de idade informaram já haver realizado exame Papanicolaou. Esse desempenho, no entanto, não produziu alteração na tendência da mortalidade, que permanece elevada. Entre as possíveis razões, devem-se considerar falhas na qualidade dos exames, falta de acompanhamento das pacientes, escassez de serviços de assistência oncológicae, sobretudo, existência de serviços inadequados e mal distribuídos ${ }^{10}$.

Em Recife, entre 2000 e 2004, a maioria dos óbitos por câncer do colo do útero ocorreu em hospitais $(85,1 \%)$, dos quais $90,2 \%$ pertenciam à rede assistencial do SUS, reforçando, desta forma, o fato de haver maior mortalidade em mulheres com mais baixa condição econômica, usuárias do sistema. Como se trata de uma morte considerada evitável, é aconselhável que se implementem programas de rastreamento eficazes, direcionados a esta população de risco, para se alcançarem níveis aceitáveis de incidência e, conseqüentemente, baixar os coeficientes de mortalidade. Um inquérito sobre a não realização do exame Papanicolaou ${ }^{19}$ mostrou que as mulheres das classes socioeconômicas mais baixas fizeram menos exames.
Segundo dados da Agência Nacional de Saúde Suplementar ${ }^{28}$, em Pernambuco, no ano de 1998, a cobertura dos planos de saúde privada era de $17,5 \%$ da população e, no ano de 2003 , caiu para $15,7 \%$ da população. Nestes mesmos anos, em São Paulo, a cobertura era de 39,2 e 38,2\% da população, respectivamente, mais do que o dobro da cobertura de Pernambuco e com uma queda proporcionalmente menor em relação aos dois anos analisados. Estes dados reforçam a baixa condição socioeconômica como fator de risco para a não realização do exame, maior incidência da doença e maior mortalidade.

Nos países em desenvolvimento, incluindo o Brasil, a prevenção do câncer do colo do útero tem caráter oportunístico, com pouco impacto na mortalidade. Cabe aos governos destes países promoverem programas permanentes de rastreamento populacional. Atualmente, pelo conhecimento que a ciência propicia para a prevenção, o câncer do colo do útero não deveria continuar como um problema de Saúde Pública.

Concluindo, as características sociodemográficas da mortalidade por câncer de colo do útero em residentes em Recife - especialmente a maior freqüência de óbitos em mulheres com idade inferior a 60 anos, negras, sem companheiro, donas de casa e residentes em bairros com baixa condição de vida, além da desigualdade no risco de morte entre os DS e da maior ocorrência em hospitais da rede assistencial do SUS - permitem a identificação de grupos prioritários de mulheres para implementação do programa de controle da doença na cidade, no intuito de alcançar coberturas de exame citológico capazes de impactar a mortalidade, com a redução de óbitos considerados evitáveis.

\section{Agradecimentos}

À Diretoria de Vigilância à Saúde da Secretaria de Saúde do Recife.

\section{Referências}

1. Derossi SA, Paim JS, Aquino E, Silva LMV. Evolução da mortalidade e anos potenciais de vida perdidos por câncer cérvico-uterino em Salvador (BA), 1979-1997. Rev Bras Cancerol. $2001 ; 47(2)$ : 163-70

2. Fonseca LAM, Ramacciotti AS, Eluf Neto J. Tendência da mortalidade por câncer do útero no Município de São Paulo entre 1980 e 1999. Cad Saúde Pública. 2004;20(1):136-42.

3. Arrossi S, Sankaranarayana R, Parkin DM. Incidence and mortality of cervical cancer in Latin America. Salud Pública Mex. 2003;45 Supl 3:306-14.
4. Ministério da Saúde. Instituto Nacional de Câncer. Estimativas 2008: incidência de câncer no Brasil [Internet]. Rio de Janeiro: Ministério da Saúde/INCA; 2007 [citado 2008 Jan 9]. Disponível em: http://www.inca.gov.br/estimativa/2008/

5. Brasil. Ministério da Saúde. Instituto Nacional de Câncer. Prevenção do câncer do colo do útero: normas e recomendações do INCA. Rev Bras Cancerol. 2003;49(4):205-6.

6. Umezulike AC, Tabansi SN, Ewunonu HA, Nwana EJ. Epidemiological characteristics of carcinoma of the cervix in the Federal capital Territory of Nigeria. Niger J Clin Pract. 2007;10(2):143-6. 
7. Linhares AC, Villa LL. Vacinas contra rotavírus e papilomavírus humano (HPV). J Pediatr (Rio J.). 2006;82(3 Supl):s25-s34.

8. Kalakun L, Bozzetti MC. Evolution of uterine cervical cancer mortality from 1979 to 1998 in the State of Rio Grande do Sul, Brazil. Cad Saúde Pública. 2005;21 (1):299-309.

9. Brasil. Ministério da Saúde. Instituto Nacional de Câncer. Periodicidade de realização do exame preventivo do câncer do colo do útero: normas e recomendações do INCA. Rev Bras Cancerol. 2002;48(1):13-5.

10. Brasil. Ministério da Saúde. Instituto Nacional de Câncer. Nomenclatura brasileira para laudos cervicais e condutas preconizadas - recomendações para profissionais de saúde. 2a ed. Rio de Janeiro: INCA; 2006.

11. Nunes J, Koifman RJ, Mattos IE, Monteiro GTR. Confiabilidade e validade das declarações de óbitos por câncer de útero no município de Belém, Pará, Brasil. Cad Saúde Pública. 2004;20(5):1262-8.

12. Antunes JLF, Wünsch-Filho V. The effect of performing corrections on reported uterine cancer mortality data in the city of São Paulo. Braz J Med Biol Res. 2006;39(8):1091-9.

13. Prefeitura Municipal do Recife. Fundação João Pinheiro. Programa das Nações Unidas para o Desenvolvimento. Desenvolvimento humano no Recife: atlas municipal [CD-ROM]. Recife; 2005.

14. Organização Mundial da Saúde (OMS). Classificação estatística internacional de doenças e problemas relacionados à saúde: $10^{\circ}$ revisão. São Paulo: Universidade de São Paulo; 1995.

15. Guimarães MB, Marques NM, Melo Filho DA, Szwarcwald CL. Condição de vida e mortalidade infantil: diferenciais intraurbanos no Recife, Pernambuco, Brasil. Cad Saúde Pública. 2003; 19(5): 1413-24.

16. Brasil. Ministério da Saúde. DATASUS [Internet]. Banco de dados sobre mortalidade 2000-2004 e categorização das variáveis em relação aos hospitais [citado 2006 Ago 28]. Disponível em http:// tabnet.datasus.gov.br/cgi/deftohtm.exe?sim/cnv/obtpe.def

17. Guerra MR, Gallo CVM, Azevedo GSM. Risco de câncer no Brasil: tendências e estudos epidemiológicos mais recentes. Rev Bras Cancerol. 2005;51(3):227-34.

18. Brasil. Ministério da Previdência Social. Aposentadoria por idade [Internet]. Brasília (DF): Ministério da Saúde; c2006 [citado
2006 Ago 15]. Disponível em: http://www.previdencia.gov.br/ pg_secundarias/beneficios_02.asp

19. Pinho AA, França Junior I, Schraiber LB, D'Oliveira AFPL. Cobertura e motivos para a realização ou não do teste de Papanicolaou no município de São Paulo. Cad Saúde Pública. 2003;19(S2): S303-S13.

20. Ng E, Wilkins R, Fung MF, Berthelot JM. Cervical cancer mortality by neighbourhood income in urban Canada from 1971 to 1996. CMAJ. 2004;170(10):1545-9.

21. Rivoire WA, Capp E, Corleta HE, Silva ISB. Bases biomoleculares da oncogênese cervical. Rev Bras Cancerol. 2001;47(2):179-84.

22. Prefeitura do Recife. Secretaria de Saúde. Diretoria de Vigilância à Saúde. Desigualdades raciais na mortalidade de residentes no Recife em 2007. Quadro de mortalidade no Recife segundo raça/ cor. Recife: Secretaria da Saúde; 2007.

23. Hackenhaar AA, César JA, Domingues MR. Exame citopatológico de colo uterino em mulheres com idade entre 20 e 59 anos em Pelotas, RS: prevalência, foco e fatores associados à sua não realização. Rev Bras Epidemiol. 2006;9(1):103-11

24. Martins LFL, Thuler LCS, Valente JG. Cobertura do exame de Papanicolaou no Brasil e seus fatores determinantes: uma revisão sistemática da literatura. Rev Bras Ginecol Obstet. 2005;27(8):485-92.

25. Leal EAS, Leal Júnior OS, Guimarães $M H$, Vitoriano MN, Nascimento TL, Costa OL. Lesões precursoras do câncer de colo em mulheres adolescentes e adultas jovens do município de Rio Branco-Acre. Rev Bras Ginecol Obstet. 2003;25(2):81-86.

26. Serra Canales I, Garcia LV, Viñales AD, Serra Canales J, Serra $\mathrm{ML}$, Zamorano CL, et al. Auditoría de muertes por cáncer cérvicouterino, Servicio de Salud Metropolitano Sur-Oriente, 1995: análisis preliminar. Rev Med Chile. 1998; 126(8):1010-8.

27. Brasil. Ministério da Saúde. DATASUS [Internet]. Siscolo: percentual de mulheres que realizaram citologia oncótica no Recife, 2000 e 2004. Brasília (DF): Ministério da Saúde; c2003. [citado 2006 Ago 15]. Disponível em: http://tabnet.datasus.gov.br/cgi/ deftohtm.exe? siscolo/DEF/todos/PECCOLO.def

28. Brasil. Ministério da Saúde. DATASUS [Internet]. Cobertura de planos de saúde em Pernambuco em 1998 e 2003. Brasília (DF): Ministério da Saúde; c2003. [citado 2006 Ago 15]. Disponível em: http://tabnet.datasus.gov.br/cgi/idb2005/f15uf.htm 